\title{
Role of gut microbiota in identification of novel TCM-derived active metabolites
}

\author{
Tzu-Lung Lin ${ }^{1}$, Chia-Chen $\mathrm{Lu}^{2,3}$, Wei-Fan Lai ${ }^{4}$, Ting-Shu Wu ${ }^{4,5,6}$, Jang-Jih Lu ${ }^{5,6}$, Young-Mao Chen ${ }^{7}$, \\ Chi-Meng Tzeng ${ }^{8}$, Hong-Tao Liu ${ }^{9}$, Hong Wei ${ }^{10}$, Hsin-Chih Lai ${ }^{1,5,6,11,12 冈}$
}

${ }^{1}$ Department of Medical Biotechnology and Laboratory Science, College of Medicine, Chang Gung University, Gueishan, Taoyuan 33302, Taiwan, China

2 Department of Respiratory Therapy, Fu Jen Catholic University, New Taipei City 24205, Taiwan, China

${ }^{3}$ Department of Chest Medicine, Internal Medicine, Fu Jen Catholic University Hospital, Fu Jen Catholic University, New Taipei City 24205, Taiwan, China

${ }^{4}$ Department of Medicine, Chang Gung University, Taoyuan 33302, Taiwan, China

${ }^{5}$ Department of Laboratory Medicine and Internal Medicine, Linkou Chang Gung Memorial Hospital, Taoyuan 33305, Taiwan, China

${ }^{6}$ Central Research Laboratory, Xiamen Chang Gung Hospital, Xiamen 361026, China

${ }^{7}$ Bachelor Degree Program in Marine Biotechnology, College of Life Sciences, National Taiwan Ocean University, Keelung 20224, Taiwan, China

${ }^{8}$ School of Pharmaceutical Sciences, Xiamen University, Xiamen 361005, China

${ }^{9}$ College of Basic Medicine, Hubei University of Chinese Medicine, Wuhan 430065, China

${ }^{10}$ Central Laboratory, Clinical Medicine Scientific and Technical Innovation Park, Shanghai Tenth People's Hospital, Tongji University, Shanghai 200435, China

${ }^{11}$ Microbiota Research Center and Emerging Viral Infections Research Center, Chang Gung University, Taoyuan 33302, Taiwan, China

12 Research Center for Chinese Herbal Medicine and Research Center for Food and Cosmetic Safety, College of Human Ecology, Chang Gung University of Science and Technology, Gueishan, Taoyuan 33303, Taiwan, China

$\triangle$ Correspondence: hclai@mail.cgu.edu.tw (H.-C. Lai)

Received March 13, 2020 Accepted May 29, 2020

\begin{abstract}
Traditional Chinese Medicine (TCM) has been extensively used to ameliorate diseases in Asia for over thousands of years. However, owing to a lack of formal scientific validation, the absence of information regarding the mechanisms underlying TCMs restricts their application. After oral administration, TCM herbal ingredients frequently are not directly absorbed by the host, but rather enter the intestine to be transformed by gut microbiota. The gut microbiota is a microbial community living in animal intestines, and functions to maintain host homeostasis and health. Increasing evidences indicate that TCM herbs closely affect gut microbiota composition, which is associated with the conversion of herbal components into active metabolites. These may significantly affect the therapeutic
\end{abstract}

Tzu-Lung Lin and Chia-Chen Lu have contributed equally. activity of TCMs. Microbiota analyses, in conjunction with modern multiomics platforms, can together identify novel functional metabolites and form the basis of future TCM research.

KEYWORDS Traditional Chinese Medicine, herbs, microbiota, transformation, multiomics

\section{INTRODUCTION}

\section{TCM and herbal formulae}

TCM has been used for centuries in China to alleviate symptoms, treat disease, and promote well-being in Chinese patients (Zhao et al., 2014). In contrast to modern Western medicine, research progress made in TCM is often inhibited because of the inherent complexity of herbs as medicine and a comparative lack of modern scientific validation. 
Accordingly, TCM research must be modernized by meeting the scientific method.

Historically, recordings that emerged from functional TCM herbal ingredients and aimed at treating specific diseases, eventually evolved into the creating of specific formulae. These formulae were further revised and assembled to create the TCM version of the "Materia Medica". These manuals established a solid basis and references of TCM for clinical treatment. Among these references, the "Shennong Bencao Jing" (literally, Shennong's Classic of Materia Medica) (Jin et al., 2013), "HuangDi NeiJing" (literally The Yellow Emperor's Classic of Medicine) (Ni, 1995), and "Compendium of "Materia Medica" (Li et al., 2014; Hao and Jiang, 2015; Gao et al., 2016; Ding et al., 2020) were featured. TCM-derived materia medicas have been rapidly developed and created complicated herbal networks for clinical applications. Each materia medica contained many formulae, with each formula comprising a combination of herbal drugs. Among these, complex components including carbohydrates/polysaccharides (PS), proteins/peptides, glycolipids/ glycoproteins, lipids, together with their metabolic derivatives such as glycosides, amines, fatty acids, flavonoids, terpenoids, phenols, and alkaloids intimately interacted with each other and modulated biological responses of immune cells and the hosts (Li and Kan, 2017; Yu et al., 2018b; Zhang et al., 2020c). Differential agonistic, compatible, or antagonistic interactions occur among TCM herbal ingredients. For instance, the Fuzheng Huayu (FZHY) is mainly composed of Radix Salvia Miltiorrhizae, Cordyceps, Semen Persicae, Gynostemma Pentaphyllum, Pollen Pini, and Fructus Schisandrae Chinensis. It is widely administered to ameliorate chronic liver diseases and functions through modulation of multiple signaling pathways in a number of organs (Chen et al., 2019). FZHY effectively regulates immune functions, optimizes systematic amino acid metabolism and endocrine function, and reduces portal vein hypertension (Chen et al., 2019). These alterations lead to improved liver function and antifibrotic effects. Additionally, FZHY also has no serious adverse reactions (Chen et al., 2019). Further, Gegen Qinlian decoction (GQD), composed of four herbs: Gegen (Radix Puerariae), Huangqin (Radix Scutellariae), Huanglian (Rhizoma Coptidis) and Gancao (Honey-fried Licorice Root) is frequently used in TCM for alleviation of type 2 diabetes (Xu et al., 2015). The Qushi Huayu Decoction (QHD), made up of Herba Artemisiae capillaris, Rhizoma Polygoni cuspidati, Herba Hyperici Japonici, Rhizoma Curcumae longae, and Gardenia jasminoides ameliorates non-alcoholic fatty liver disease (NAFLD) in patients (Feng et al., 2013).

\section{Current advances in identification of active components from TCM herbs}

The basic principle of scientific exploration in Western medicine has been the discovery of functional compounds and their corresponding targets in specific signaling pathways within cells. To achieve this understanding, standardized phytochemistry, pharmacology, pharmacokinetics (PK, absorption/distribution/metabolism/excretion, ADME), pharmacodynamics (PD, effects/action/mechanism), and toxicology research procedures are performed (Chen et al., 2020). Currently, platforms for high throughput screening of compounds, together with stringent functional and safety validations are used to better understand the mechanisms of action of functional compounds. Concordantly, for the development of novel therapeutic drugs from TCM-derived herbs, a similar approach was established (Martel et al., 2017a; Jiang et al., 2020). Through this method, many active components in TCM herbs were identified. One famous example was the discovery of artemisinin, a plant-derived compound with anti-malaria and anti-cancer functions (Zhang et al., 2007; Carqueijeiro et al., 2019). Other examples included berberine purified from berberis, capsaicin from chili peppers, caffeine from coffee beans, ephedrine from Ephedra, chitosan from mushrooms, genistein from soybeans, celastrol from thunder god vine, epigallocatechin gallate from green tea, glycyrrhizin from licorice roots, quercetin from various plants, and curcumin from turmeric (Martel et al., 2017b). Besides small chemicals, functional polysaccharides (PS) derived from TCM herbs have also been characterized, such as those from Ganoderma lucidum mycelium (Chang et al., 2015), Hirsutella sinensis mycelium (Chang et al., 2015; Wu et al., 2019), and Poria cocos (Sun et al., 2019).

To improve the efficiency of screening for novel functional TCM herbal components, new approaches using modern technology have been explored. For example, a luciferasebased high-throughput screening (HTS) assay has been used to integrate multiple chemical messages derived from effective TCM healing formulae. This pipeline can expedite the active ingredient discovery process by reducing replicated leads (Yu et al., 2019a). Beyond this screen, a TCM System Pharmacology Database and Analysis Platform (TCMSP) had been established. The TCMSP is a systematic pharmacology database which compiles drug discovery results from previous herbal medicine experiments. This database contains pharmacochemistry, ADME and toxicity properties, drug likeness and targets, associated diseases, and interaction networks. Importantly, this database can be used to unravel active components in TCM herbs and their targeted cellular pathways (Ru et al., 2014; Li et al., 2020). Exploration of this database can be combined with other systems, such as Gene Ontology (GO) predictions and Kyoto Encyclopedia of Genes and Genomes (KEGG) pathway enrichment analyses to identify potential ameliorative mechanisms of key molecules (Yu et al., 2019a). Additionally, interactions between active molecules and their predicted target proteins may be further predicted by "molecular docking" and protein-protein interaction networks, which may enhance understanding of underlying potential interactions. Finally, the TCMSP database enables the linking of identified 
compounds to their corresponding targets/pathways involved in disease amelioration. Therefore, through these analyses, axis of component-target-disease (C-T-D) and the corresponding target-pathway (T-P) networks could be established, to further dissect the active compounds, potential targets, and core pathways in treatment of diseases by a specific TCM formula (Li et al., 2020). Subsequently, LC and/or GC-MS/MS can be used to practically monitor the active ingredients of TCMs. This style of pipeline may provide a new, standardized approach to systematically screen TCM herbal components for treatment of diseases ( $\mathrm{Li}$ et al., 2020). Accordingly, functional molecules in TCMs can be assessed in the context of heterogeneous cell signaling pathways to predict their effects on diseases at immunological, metabolic, and molecular levels (Zhang et al., 2016; Li and Kan, 2017).

Even though many in vitro assay-based screening systems are available for high throughput screening, most purified small chemical components directly derived from herbs still suffer from marginal potency, adverse effects, and low bioavailability in animal or clinical studies (Belcher et al., 2019; Liu et al., 2019a; Teijaro et al., 2019). For example, the compound rhein showed beneficial effects on diabetic nephropathy, which is related to reduced levels of TGF- $\beta_{1}$, renal fibrosis, metabolism, and oxidative stress status (Hu et al., 2019). However, its adverse effects, such as hepatotoxicity, nephrotoxicity, and embryonic toxicity were also highlighted (Yuan et al., 2016). Further, chemical compounds derived from Polygonum multiflorum (also known as Heshouwu) showed ameliorative effects on hair-blackening, liver and kidney-tonifying, anti-aging, as well as neuronal disease treatment (Lin et al., 2015). However, these compounds could induce hepatotoxicity, nephrotoxicity and embryonic toxicity (Lin et al., 2015). Comparatively, the PS purified from TCM herbs showed less toxicity and were frequently modified in the host (Chen et al., 2016). So far the underlying molecular mechanism of PS effects remains poorly understood.

\section{GUT MICROBIOTA MAINTAINS INTESTINAL HOMEOSTASIS AND PROMOTE HEALTH}

The gut microbiota is a collection of microbes colonizing the intestine (Lin et al., 2014; Tsai et al., 2019; Zmora et al., 2019). More than 100 trillion $\left(10^{14}\right)$ microbes inhabit the human gastrointestinal (GI) tract, which included about 10 times more bacterial cells than the number of human cells, and over 100 times the amount of genetic contents (microbiome) in contrast to the human genome (Thursby and Juge, 2017). Bacteria at the number between $10^{2}-10^{4}$ colonyforming units (CFU)/mL are found in the first section of the small intestine, the duodenum. Generally, the Lactobacilli, Streptococci, Veillonellae, Staphylococci, Actinobacilli and yeasts dominate the duodenum and jejunum (Lin et al., 2014). The GI microbiota changes markedly from the duodenum to the ileum, with an according increase in bacterial load, reaching up to $10^{6}-10^{8} \mathrm{CFU} / \mathrm{mL}$. In the large intestine, commensal bacteria reach high numbers $\left(10^{7}-\right.$ $10^{12} \mathrm{CFU} / \mathrm{mL}$ ) and are extremely diverse. At the same time, the environment of colon is strictly anaerobic. This means that obligate anaerobes that obtain their energy from fermentation dominate. More than 1,500 bacterial species have been identified in the colon of humans (Lin et al., 2014; Chang et al., 2019). However, a large proportion of the GI microbiota bacteria cannot be easily isolated and cultured in vitro, necessitating the use of culturomics (Lagier et al., 2018).

Under homeostasis situation, the intestinal microenvironment provides a barrier to prevent the translocation of pathogens or harmful agents (such as the endotoxin LPS) across the intestinal epithelial cells (IECs) into the surrounding lymphoid system and blood (Boyapati et al., 2016). Gut microbiota bacteria maintain host integrity and regulate many important physiological functions, including homeostasis of energy and metabolism, modification of xenobiotics, modulation of intestinal homeostasis, regulation of immunity and protection against pathogens, and even normal host neuronal behavior and cognitive functions (Clemente et al., 2012; Schachter et al., 2018; Lin et al., 2019).

In aberrant physical, chemical, or biological conditions, such as long-term high fat diet, antibiotic treatment, or increased oxidative stress, the composition of gut microbiota changes, lead to $\mathrm{Gl}$ dysbiosis and disruption of the intestinal mucosa. This dysbiosis results in a damaged intestinal barrier and increased intestinal permeability. Proinflammatory elements, such as pathogen-associated molecular pattern molecules (PAMPS) (mainly the endotoxin, LPS) or damage-associated molecular pattern molecules (DAMPS) (Tang et al., 2012), are increased in intestines and blood, resulting in both local intestinal injury and systemic chronic inflammation (Yan, 2018; Alexandrov et al., 2019). This "leaky gut" phenomenon is closely related to development of chronic inflammation-related diseases. In a broad sense, the gut microbiota appears to be critical in maintaining host homeostasis and health (Lin et al., 2014; Wang et al., 2017a; Gentile and Weir, 2018).

\section{CLOSE INTERACTION BETWEEN TCM HERBS AND GUT MICROBIOTA IN DISEASES AMELIORATION}

TCM herbs closely interact with gut microbiota and affect their composition (Peng et al., 2020). Reciprocally, the gut microbiota also plays essential roles in the conversion of carbohydrates, proteins, lipids, and non-nutritive small chemical compounds from TCM herbs into chemical metabolites that may show beneficial or adverse effects on human health (Blaut and Clavel, 2007; Wang et al., 2013; Yu et al., 2018a; Feng et al., 2019; Lu et al., 2019; Qu et al., 2019; Yue et al., 2019; Zhang et al., 2019a, b, 2020c) (Fig. 1 
and Table 1). These results indicated that modulation of gut microbiota composition may contribute to the effects of disease amelioration by TCM treatment. For oral treatment, TCM herbs have mostly been prepared by soaking the ingredients in boiling/hot water to generate a water extract that contains a mixture of chemical components, which was generally named as "decoction" (Zhou et al., 2016; Chi et al., 2019; Deng et al., 2019). While some TCM nutraceuticals may directly affect epithelial and immune cells of the digestive tract; others, such as indigestive PS, polyphenols, and alkaloids, etc., may pass through the stomach and reach small and large intestine. Many herbal ingredients are frequently fermented or converted by local gut microbiota to form bioactive, bioavailable, or even toxic metabolites (Lyu et al., 2017; Liu et al., 2018b; Dey, 2019; Wu and Tan, 2019; Yang and Lao, 2019). Depending on use of different formulae, some transformed metabolites may be functionally novel and not clearly defined (Fig. 1). Both changed microbiota bacteria and transformed TCM metabolites may contribute to control of progression of diseases development. Take the TCM effects on amelioration of diabetes as an example: while almost all bacterial phyla seemed to be affected by
TCM herbs administration, Bacteroidetes, Firmicutes (and therefore the Firmicutes/Bacteroidetes (F/B) ratio), Proteobacteria, Verrucomicrobia, Cyanobacteria, Deferribacteres, and Actinobacteria were mostly reported (Dey, 2019; Zhang et al., 2019a). Further detailed analyses indicated the abundance of potentially beneficial (such as anti-inflammatory, or SCFAs producers) and harmful (proinflammatory and pathogenic) bacteria could be differentially affected by ingestion of different TCM herbs (Chang et al., 2015; Lyu et al., 2017; Tong et al., 2018; Lin et al., 2019; Nie et al., 2019; Wu et al., 2019). Alteration of the gut microbiota composition is therefore closely related to development of differential immune and metabolic activities in the hosts. Roles of these bacteria on health or disease development are species or even strain dependent under different disease situations, which is under intensive study (Lin et al., 2019).

Recent studies have shown a variety of TCM herbal components influence microbial abundance and diversity, which reciprocally is closely related to the efficacy of TCM herbs (Table 1). Among these, effects from PS treatment on obesity, diabetes and related metabolic syndromes seemed to be most intensively studied. For example, treatment with

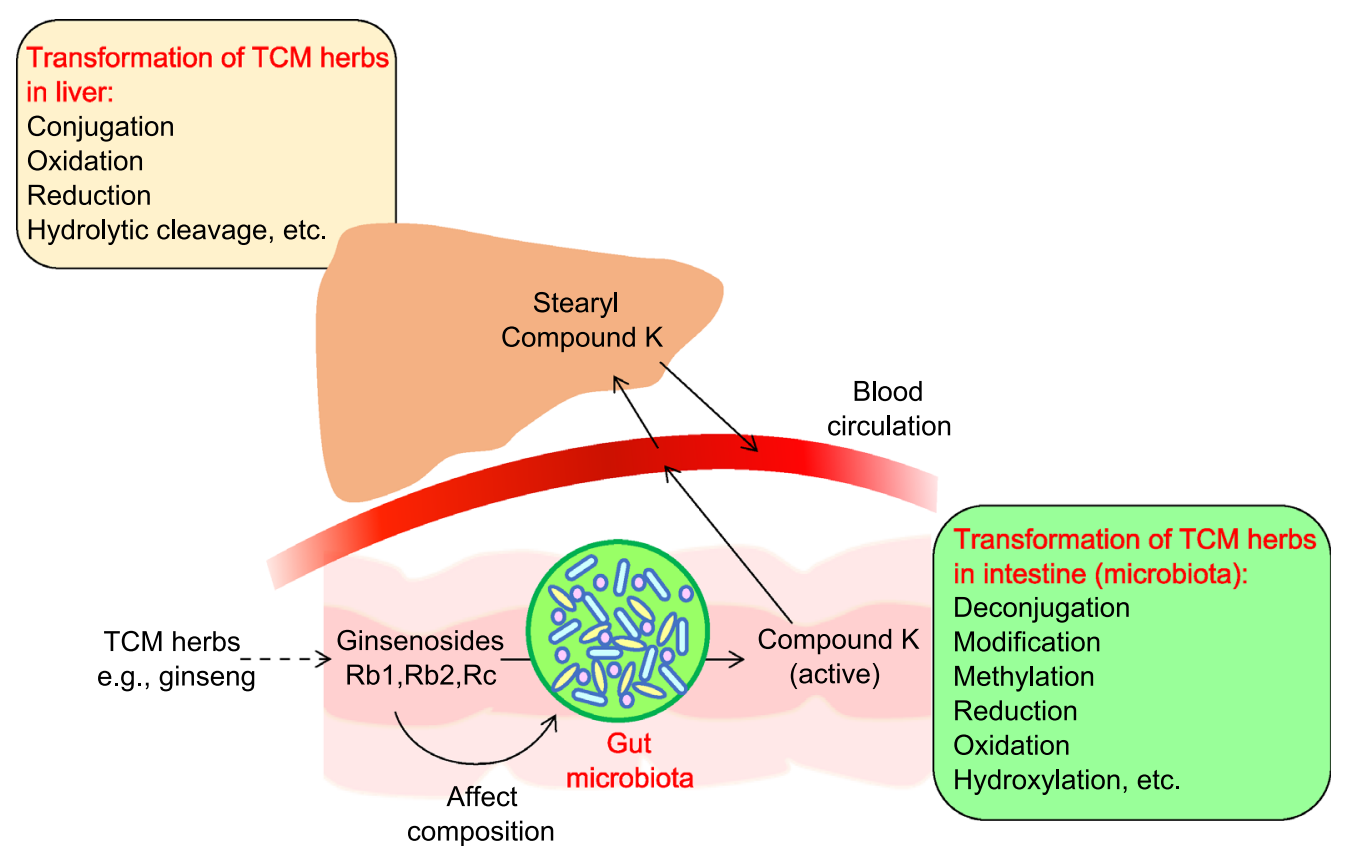

Figure 1. The transformation of TCM herbal ingredients (ginseng extracts as an example). Transformation of TCM herbal ingredients into active metabolites in host was contributed both by gut microbiota and liver. Understanding ginseng's pharmacokinetics is important for better medication in patients. After oral administration of ginseng, the bioavailability of ginsenosides is low, and the metabolites transformed by gut microbiota may become biologically active. For example, ginsenosides $\mathrm{Rb} 1, \mathrm{Rb} 2$ and Rc were transformed by gut microbiota to form compound K, followed by absorption into the blood (Qi et al., 2011). Compound $\mathrm{K}$ showed higher potency and activity compared with ginsenoside $\mathrm{Rb} 1$. Compound $\mathrm{K}$ adsorbed into blood metabolized again in liver to form stearyl compound $\mathrm{K}$ (Kim, 2018). On the other hand, ginsenoside Rb1 also could modulate the composition of gut microbiota (Wan et al., 2017). Therefore, gut microbiota produces active metabolites and plays an important role in the pharmacological action of orally administered ginseng. 


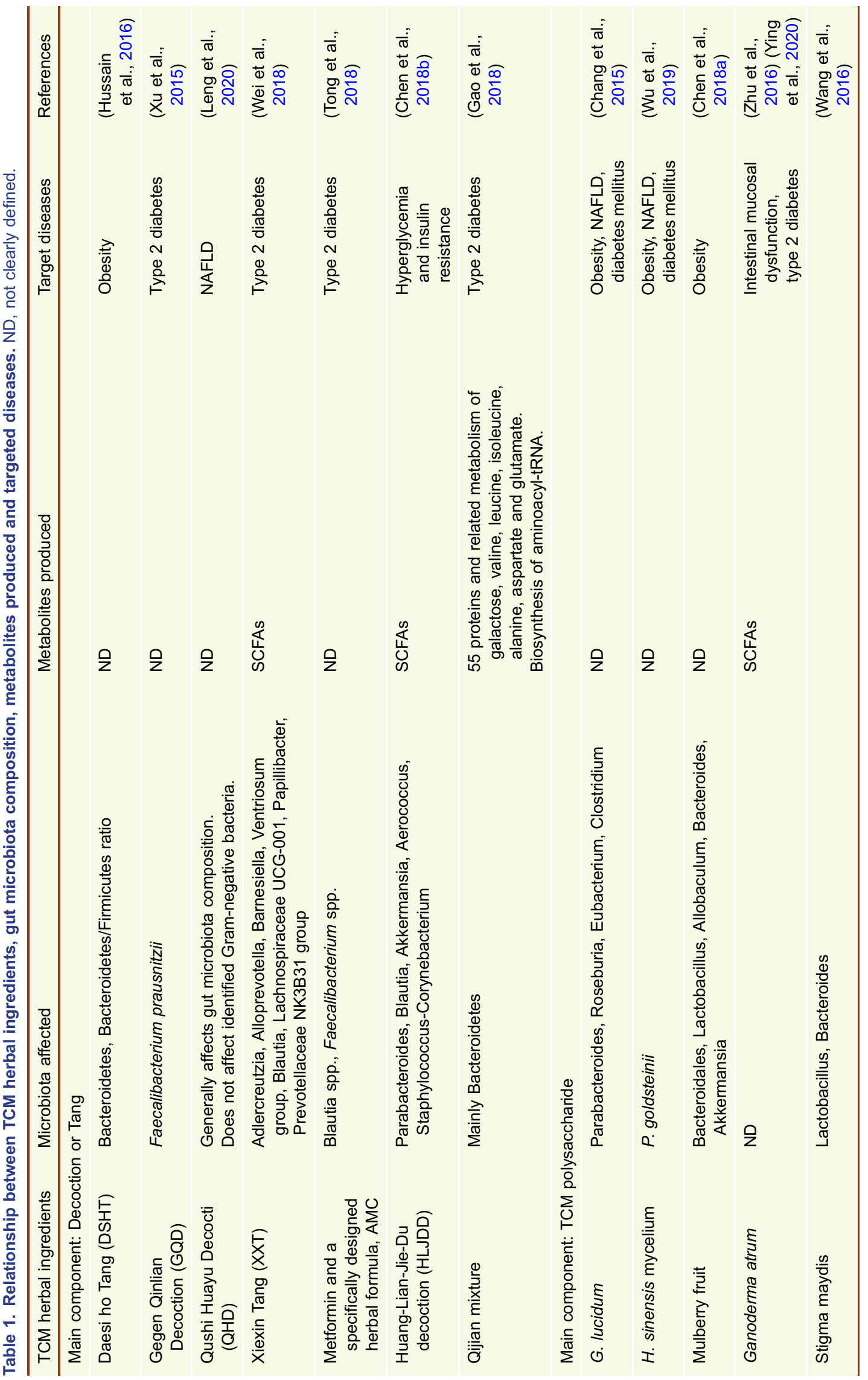




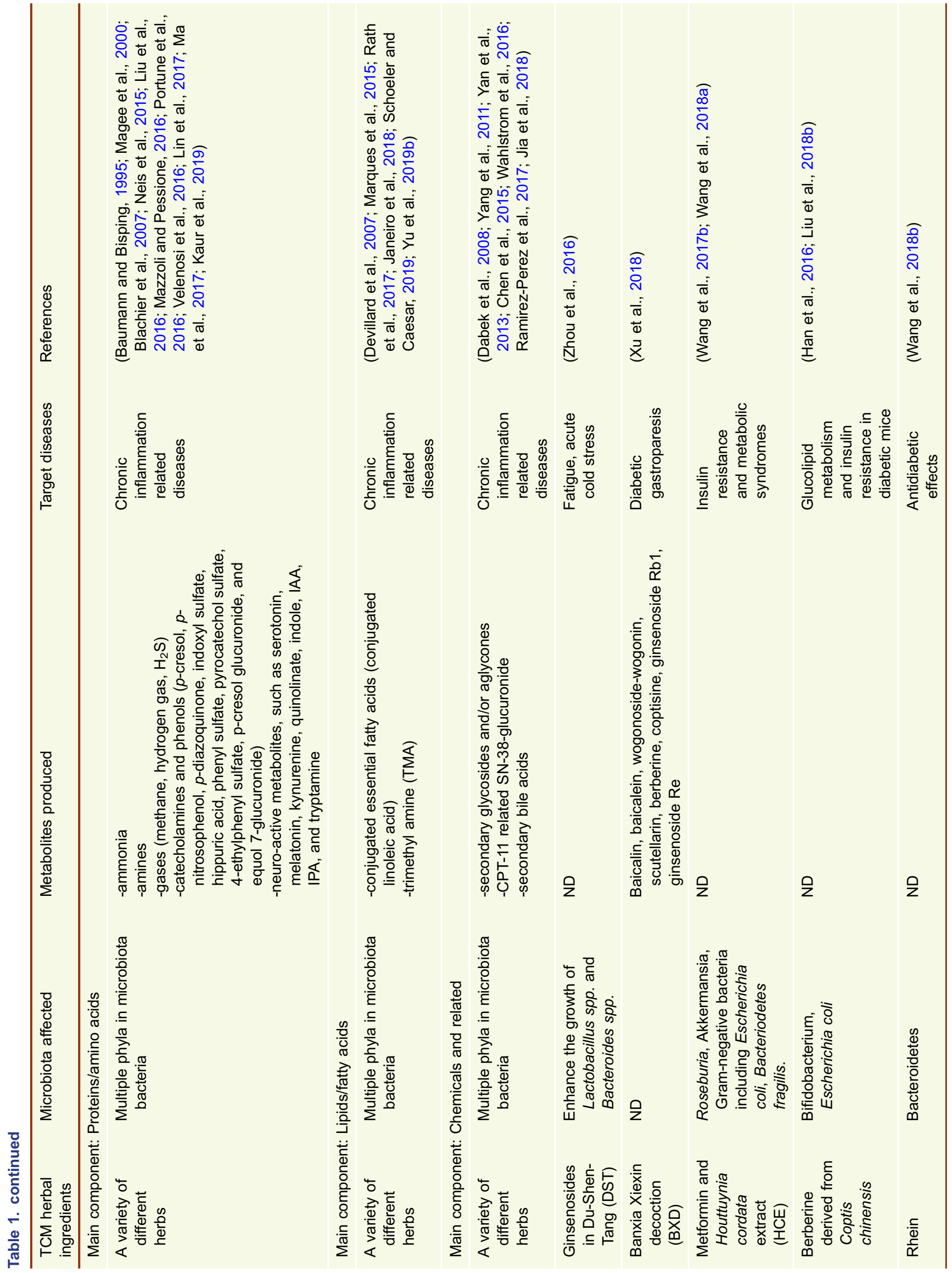


PS purified from $G$. lucidum and $H$. sinensis mycelium significantly reduced obesity through enhancement of a consortium of commensal bacteria, including Parabacteroides goldsteinii, Roseburia, Eubacterium and Clostridium spp. (Chang et al., 2015; Wu et al., 2019). Mulberry fruit PS also influenced obesity and modulated gut microbiota (Chen et al., 2018a), while PS from Ganoderma atrum ameliorated intestinal mucosal dysfunction and improved liver function in type 2 diabetes (Zhu et al., 2016), and stigma maydis PS ameliorated type-2 diabetes (Wang et al., 2016) by changing gut microbial populations and related fermentation functions. Further, the PS and ginsenosides contained in decoction of ginseng, the Du-Shen-Tang (DST), restored fatigue and acute cold stress, and modulates the gut microbiota composition (Zhou et al., 2016). There were many other examples highlighting the microbial change during the treatment of obesity, diabetes and metabolic syndromes (Table 1). These examples include Daesiho-Tang (DSHT) that attenuated obesity and significantly increased the relative abundance of Bacteroidetes, B/F ratio, Akkermansia, Bifidobacterium, Lactobacillus, and decreased the level of Firmicutes (Hussain et al., 2016), Gegen Qinlian Decoction (GQD) alleviated Type 2 diabetes and significantly increased Faecalibacterium prausnitzii (Xu et al., 2015), while Qushi Huayu Decoction (QHD) reduced HFD-induced non-alcoholic fatty liver disease (NAFLD), and significantly increased the abundance of Parabacteroides and decreased the abundance of Odoribacter, Rikenella, Tyzzerella, Intestinibacter, Romboutsia and 2 members in Lachnospiraceae (Leng et al., 2020). Other examples related to gut microbiota changes included (Table 1): $20 \%$ Folium Mori amelioration of diabetes (Zhang et al., 2019a), Xiexin Tang-mediated improvement of type 2 diabetes (Wei et al., 2018), amelioration of human type 2 diabetes by metformin and a traditional Chinese herbal formula, AMC (Tong et al., 2018), Huang-Lian-Jie-Du decoction-mediated treatment of hyperglycemia and insulin resistance (Chen et al., 2018b), improvement of type 2 diabetes by treatment with Qijian (Gao et al., 2018), Banxia Xiexin decoction on diabetic gastroparesis rats (Xu et al., 2018), Houttuynia cordata facilitation of metformin on reducing insulin resistance (Wang et al., 2017b), berberine, the main bioactive alkaloid of Coptis chinensis, on glucolipid metabolism and insulin resistance in diabetic mice (Han et al., 2016; Liu et al., 2018a), and rhein's role in antidiabetic effects (Wang et al., 2018b). Evidently, efficacy of TCM herbal treatment is closely related to their influence on gut microbiota composition. Therefore, the ingredients of TCM herbs may not only directly regulate host cells activity, but also be metabolized by gut microbiota and affect their structures. Close interaction between TCM and gut microbiota contributes to modulating the host immune and metabolic activities. New research approaches based on gut microbiota-related characterization of clinically applicable TCM components have to be developed (Zhao et al., 2014).

\section{ROLE OF GUT MICROBIOTA IN FERMENTATION OF TCM PS}

TCM PS as well as other plant-derived PS such as vegetables, fruits, and whole grains etc. were shown to play important functions in immune-modulation and disease amelioration (Chang, 2002; Yang et al., 2009; Li et al., 2013; Yu et al., 2018b; Sun et al., 2019). For example, both high (>100 kDa.) and low (<100 kDa.) molecular weight, and neutral and acidic PS prepared from Panax ginseng that displayed differential functions affecting cellular and host physiology (Sun, 2011; Kim et al., 2020) were among the many examples. Owing to the limited digestive enzymes encoded by the human genome, PS are frequently undigested until they reach the colon (Baumann and Bisping, 1995). In the colon, fermentable PS, such as $\beta$-glucans, were broken down by gut microbes via their saccharolytic machinery to produce important groups of natural bioactive products. Serial digestion of PS resulted in production of a number of short-chain oligosaccharides, comprising varying chain lengths, structural conformations, and number of branches (Tzianabos, 2000; Santa et al., 2014). These varied oligosaccharides may function to enhance growth of probiotics, such as Bifidobacterium spp. and Bacteroides spp. (Bouhnik et al., 2004). The shorter PS may be further digested to form either monosugars such as mannose (Man), glucose (Glc), galactose (Gal), rhamnose (Rha), arabinose (Ara), and fucose (Fuc) which enhance bacterial growth (Koropatkin et al., 2012; Tremaroli and Backhed, 2012). Monosugars can continuously be catabolized to form metabolites such as short chain fatty acids (SCFAs) (formate, acetate, propionate, butyrate), lactate, hydrogen, and carbon dioxide, which may directly affect host physiology (Schwiertz et al., 2010; Simpson and Campbell, 2015; Burokas et al., 2017; Martel et al., 2017a). On the other hand, degraded products such as D-mannose may act as signaling molecules which show differential immunomodulatory effects and functions towards host cells and tissues (Zhang et al., 2017; Zhang et al., 2018).

A consortium of gut bacteria participates in the degradation of TCM-derived PS. For example, a host of bacterial species are involved in butyrate production, including F. prausnitzii, Eubacterium rectale, Roseburia spp. Clostridium spp. and many others belonging to Bacteroidetes (Zhang et al., 2018). These functional bacteria were characterized by their displays of several to dozens of enzymes used to degrade PS by targeting specific glycosidic linkages or chemical substituents (Martens et al., 2011). Genetic clusters involved in binding, degradation and importation of various PS include miscellaneous polysaccharide utilization loci (PULs), or starch utilization system (Sus)-like systems in Bacteroidetes (Bayer et al., 2008; Ravcheev et al., 2013). Comparatively, with respect to carbohydrate active enzymes (CAZymes), Bacteroidetes degrade a relatively wide range of polysaccharides, while Firmicutes prefer to catabolize selected polysaccharides (Cockburn and Koropatkin, 2016; 
Zhang et al., 2018). Many different bacterial species may involve sequential catabolism of PS, and the functional metagenomics of consortium of bacteria that participate in PS metabolism are currently under intensive study.

\section{TCM AFFECTS GUT MICROBIOTA THAT PRODUCES FUNCTIONAL AMINO ACIDS METABOLITES}

Dietary proteins including those derived from TCM herbs, dietary foods, and other nutraceuticals can be digested by both hosts and gut microbiota, which may further affect gut microbiota structure (Conlon and Bird, 2014; Madsen et al., 2017; Amaretti et al., 2019). At the same time, certain gut bacteria in the distal colon also metabolize amino acids to form unique functional metabolites through specific biochemical pathways (Baumann and Bisping, 1995; Neis et al., 2015; Liu et al., 2016). The metabolic intermediates produced may involve either optimal modulation of energy homeostasis, nutrition metabolism, intestinal health and immunity, or cause inflammation and diseases (Neis et al., 2015; Portune et al., 2016; Lin et al., 2017). Basically, deamination of amino acids results in the production of ammonia, whereas decarboxylation leads to amine production (Baumann and Bisping, 1995). Depending on the amount of proteins up taken, the concentrations of ammonia, trace amines, and gases (methane, hydrogen, $\mathrm{H}_{2} \mathrm{~S}$ ) related to cytotoxins, genotoxins, and carcinogens produced in colon are altered (Ma et al., 2017). These harmful metabolites may translocate across the intestinal barrier and enter the blood for systematic circulation, resulting diseases development.

Anaerobic fermentation of cysteine and methionine by bacteria results in $\mathrm{H}_{2} \mathrm{~S}$ formation. Long term increased sulfide and ammonia concentrations in the colon were shown to promote colitis and tumorigenesis (Baumann and Bisping, 1995; Magee et al., 2000). Also, metabolism of aromatic amino acids may produce a group of uremic toxins, including indoxyl sulfate, p-cresyl sulfate, hippuric acid, phenyl sulfate, pyrocatechol sulfate, 4-ethylphenyl sulfate, p-cresol glucuronide, and equol 7-glucuronide (Velenosi et al., 2016). These compounds are closely related to the development of chronic kidney disease (CKD), where the gut-plasma-kidney metabolic axis is established (Mishima et al., 2017; Liu et al., $2018 b)$. Furthermore, degradation of "tyrosine" gives rise to catecholamines and phenols, where $p$-cresol, $p$-nitrosophenol and $p$-diazoquinone are proposed to show carcinogenic effects (Bone et al., 1976; Kikugawa and Kato, 1988), tyramine is related to hypertension, and DOPA, dopamine and nor-adrenaline can modulate multiple physiological functions (Mazzoli and Pessione, 2016). On the other hand, the anaerobic conversion of "tryptophan" also produces functional metabolites related to the gut-brain axis (GBA). These include neuro-active metabolites, such as serotonin, melatonin, kynurenine, quinolinate, indole, IAA, IPA, and tryptamine (Mazzoli and Pessione, 2016; Kaur et al., 2019). Other neuroactive molecules including histamine, glutamate, and GABA are also synthesized owing to the close interaction between the host and the microbiota (Sharon et al., 2014; Mazzoli and Pessione, 2016). The gut bacteria involved in such metabolism processes were widely categorized into five phyla including Actinobacteria, Firmicutes, Bacteroidetes, Proteobacteria, and Fusobacteria, in which Clostridium, Burkholderia, Streptomyces, Pseudomonas, and Bacillus were further enriched to be involved in the many tryptophan metabolism pathways (Kaur et al., 2019).

Branched chain amino acids (BCAAs), such as leucine (Leu), isoleucine (lle), and valine (Val), work both as the substrates for anabolism of nitrogenous compounds and as signaling molecules regulating energy homeostasis via multiple signaling networks, including the phosphoinositide 3-kinase/protein kinase $\mathrm{B} /$ mammalian target of rapamycin (PI3K/AKT/mTOR) pathway (Nie et al., 2018). Gut microbiota also participates in fermentation of BCAA and generates a complex mixture of metabolites, including ammonia, SCFAs, and branched-chain fatty acids (valerate, isobutyrate, and isovalerate). These bacterial metabolites have been shown to influence normal mucosal immunity of the host (Blachier et al., 2007).

Generally, the abundance of gut microbiota bacteria that are involved in amino acids metabolism are frequently affected by TCM herbs administration (Chang et al., 2015; Lyu et al., 2017; Tong et al., 2018; Feng et al., 2019; Lin et al., 2019; Nie et al., 2019; Wu et al., 2019; Yue et al., 2019; Zhang et al., 2019a). These included the Clostridium clusters, Bacillus, Lactobacillus, Streptococcus, and Proteobacteria in human small intestine, and the Clostridia and Peptostreptococci in large intestine of healthy humans (Neis et al., 2015). More and more bacterial species are expected to be unraveled to be involved in protein metabolism. Therefore, advances with regard to unraveling the protein/amino acid fermentation pathways, the potential novel corresponding metabolites produced by gut microbiota, and modulation of microbiota composition by TCM herbs are essential (Zhang et al., 2019b).

\section{TCM-AFFECTED GUT MICROBIOTA PRODUCES FUNCTIONAL METABOLITES IN LIPID METABOLISM}

Lipids and their derived functional metabolites play multiple physiological roles in the host. Lipids from TCM herbs and daily foods intake have also been shown to affect gut microbial growth and composition, while gut microbiota bacteria are also important players in lipid metabolism in hosts (Marques et al., 2015; Di et al., 2019; Schoeler and Caesar, 2019; Yu et al., 2019b) (Table 1). Besides SCFAs, many other functional metabolites derived from lipid metabolism are also produced by bacteria in the gut. For example, commensal bacteria, including Roseburia, Lactobacillus, Butyrivibrio, and Megasphaera participate in fatty acid metabolism to produce an array of conjugated essential fatty 
acids (e.g., conjugated linoleic acid) that intricately influence host physiology (Devillard et al., 2007). On the other hand, milk fat increases taurine-conjugation of bile acids, which leads to the further growth of Bilophila wadsworthia that then uses the increased availability of amino acid-derived sulfur to produce $\mathrm{H}_{2} \mathrm{~S}$ closely related to the incidence rates of colitis (Devkota et al., 2012).

Using key enzyme components of the trimethylamine (TMA)-synthesis pathways such as the choline TMA-lyase (CutC) pathway and carnitine oxygenase (CntA) pathway, gut bacteria such as Clostridium XIVa strains and Eubacterium spp. catabolize choline and L-carnitine to produce TMA. TMA is converted to trimethylamine- $N$-oxide (TMAO) in the liver (Rath et al., 2017; Janeiro et al., 2018). Many studies have indicated a close association between TMAO plasma levels and the risk of atherothrombotic cardiovascular disease (CVD) (Canyelles et al., 2018).

Previous studies have highlighted the roles of gut bacteria, such as Akkermansia, Butyricimonas, Christensenellaceae, Eggerthella, Tenericutes, and Pasteurellaceae, on affecting specific aspects of lipid metabolism and/or distinct classes of lipoproteins (Ghazalpour et al., 2016). At the same time, rapid progresses have been made in unraveling the underlying mechanisms of TCM-mediated regulation of lipid metabolism and amelioration of disease. For instance, TCMs have been shown to inhibit intestinal absorption, reduce de novo biosynthesis, increase catabolism, and enhance secretion in lipids (Bei et al., 2012). The changed gut microbiota composition by TCM herbs administration may contribute to the ameliorative effects on abnormal lipids metabolism (Huang et al., 2019; Zhang et al., 2019a). On the other hand, ginseng extract can enrich Enterococcus faecalis that produces an unsaturated long chain fatty acid, myristoleic acid (MA), leading to reducing adiposity by activation of brown adipose tissue (BAT) and formation of beige fat (Quan et al., 2019). Therefore, modulation of gut microbiota bacteria by TCM herbs may produce optimal amounts of beneficial lipid metabolites, which is an effective strategy for promotion of well-being in hosts.

\section{TRANSFORMATION OF TCM SMALL CHEMICAL MOLECULES BY GUT MICROBIOTA}

Different TCM formulae contain an array of small chemical molecules with a number biological functions, including flavonoids, saponins, alkaloids, and anthraquinones, etc. (Xu et al., 2013; Yan et al., 2013; Chen et al., 2015; Zhang et al., 2020b) (Table 1). Both gut microbiota and the liver involve conversion of the xenobiotics ingredients into subsequent metabolites (Fig. 1). In the intestines, these components can be modified/deconjugated by gut microbiota. Alternatively, they can also be absorbed and transported to the liver, where they are also modified/conjugated to increase their water solubility and facilitate excretion. After excretion into the intestinal tract from liver, these metabolites may further undergo modification/deconjugation by gut microbiota to form secondary metabolites (Wahlstrom et al., 2016; Kim, 2018). Many of the transformed metabolites frequently act as functional compounds directly influencing the curative effects of TCM treatment. For example, herbal glycosides such as saponins, geniposide, iridoid glycosides, and flavone glycosides frequently identified in TCM ingredients are metabolized into secondary glycosides or aglycones by bacterial $\beta$-glucosidase (GUS) (Dabek et al., 2008; Yang et al., 2011) (Fig. 1). Such transformation may affect the bioavailability and bioactivity of these molecules (Yang et al., 2011). Additionally, the gut microbial GUS enzymes encoded by a variety of gut bacteria belonging to Firmicutes $(60 \%)$ and Bacteroidetes (21\%) (Humblot et al., 2007; Creekmore et al., 2019) also catalyze hydrolysis of $\beta$-D-glucuronic acid from their conjugated compounds and influence drug potency and toxicity. The transformation of ginseng extracts is taken as an example (Fig. 1). After oral administration, ginsenosides such as $\mathrm{Rb} 1, \mathrm{Rb} 2$ and $\mathrm{Rc}$ from ginseng extracts were transformed by gut microbiota to form $20-0-\beta$ D-glucopyranosyl-20(S)-protopanaxadiol (compound $\mathrm{K}$ ) which was absorbed into the blood to achieve the pharmacological functions. Compound $\mathrm{K}$ showed more potent antitumor, anti-inflammatory, and anti-allergic activities more than ginsenoside Rb1 (Wang et al., 2011; Kim et al., 2013). Therefore, the gut microbiota plays an important role in the pharmacological action of orally administered ginseng.

Another example is the CPT-11 which is a potent anticancer agent metabolized to the active compound, SN-38 in vivo. A balanced $\mathrm{SN}-38$ concentration in patients is essential to maintain optimal efficacy of cancer treatment while reducing toxicity. Intriguingly, both TCM components and gut microbiota are involved in modulation of $\mathrm{SN}-38$ activity. For detoxification, SN-38 is conjugated to SN-38glucuronide by UDP-glucuronosyltransferase (UGT). However, purified herbal aglycons where the glycosyl group of a glycoside was removed inhibited UGT activity (Yokoi et al., 1995; Ramesh et al., 2010; Bailly, 2019), leading to an increase in toxic SN-38 concentration in the enterohepatic circulation. On the other hand, gut microbiota bacteria also use the gus encoded $\beta$-glucuronidase for deconjugation of the $\mathrm{SN}$-38-glucuronide. Therefore, the underlying regulatory activities formed a complicated regulatory network (Dabek et al., 2008). How to achieve a balanced SN-38 activity in patients with maximal efficacy of cancer treatment while reducing toxicity is an important issue.

Gut microbiota also transform bile acids and cholesterols, producing a variety of functional metabolites (Gerard, 2013). Among these, primary bile acids are produced in the liver as glycine, taurine, or sulfate conjugates, and after secretion into the intestine, are deconjugated and modified by intestinal bacteria. Through interaction with bile acid farnesoid $X$ receptor $(F X R)$ and $G$ protein-coupled bile acid receptor 1 (TGR5), bile acids signaling controls multiple important physiological behaviors and maintains intestinal homeostasis and a healthy environment (Jia et al., 2018). Bacteria such as 


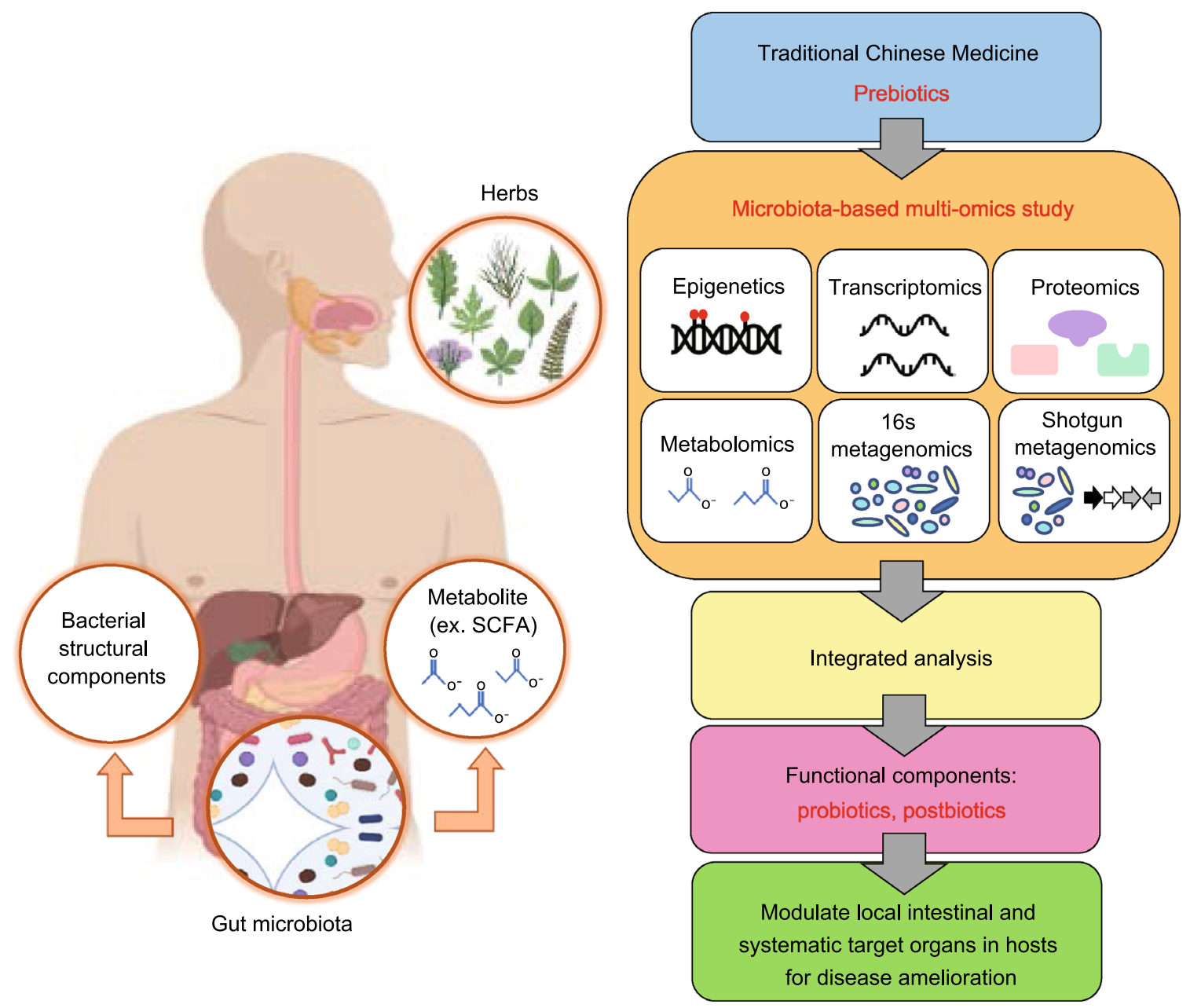

Figure 2. Microbiota-based integrated multi-omics platforms for TCM-derived herb study. The basic rationale is to identify TCM herbs related active components after transformation by gut microbiota fermentation. The multiomics platforms such as, epigenetics, 16s metagenomics, shotgun metagenomics, transcriptomics, proteomics, and metabolomics are to be used, followed by the integrated analysis. Such strategy may identify novel TCM prebiotics, bacteria (probiotics) and metabolites (postbiotics) as well as bacterial structural components (paraprobiotics) to modulate local intestine and systematic target organs in hosts for disease amelioration.

Bacteroides, Bifidobacterium, Clostridium, Egghertella, Escherichia, Eubacterium, Fusobacterium, Lactobacillus, Listeria, Peptococcus, Peptostreptococcus, Pseudomonas, and Ruminococcus, use their enzymes in deconjugation, oxidation and epimerization, 7-dehydroxylation, esterification, and desulfatation of the bile acids (Gerard, 2013). Secondary bile acids that might work as tumor promoters are therefore produced after bacterial fermentation in intestine (Wahlstrom et al., 2016; Ramirez-Perez et al., 2017; Jia et al., 2018). Accordingly, the development of disease-treatment strategies using TCM herbs, or use of metabolically engineered bacteria to modify chemicals for maintaining health are warranted (van Duynhoven et al., 2011; Lee et al., 2012; Anlu et al., 2019).

\section{MICROBIOTA-BASED INTEGRATED MULTIOMICS STUDY-THE NEXT GENERATION TCM HERBAL RESEARCH}

Owing to the difficulties encountered in TCM research, one must consider developing novel strategy to characterize TCM herbs related active components. For future TCMderived herbal studies, use of microbiota-based integrated multiomics platforms seems critical (Fig. 2). The underlying basic rationale is as follows: instead of directly screening functional components from herbal extracts, identifying functional components after transformation by gut microbiota fermentation using multiomics approaches (Fig. 2). Prepared TCM herbal products (such as crude extracts/decoctions or powders), are first fed to animals to evaluate their efficacy of 
disease amelioration. If positive results are obtained, the classification and abundance of bacteria in intestine/feces, and their derived metabolites (including bacterial structural components containing potential paraprobiotics) in intestine/ feces/blood samples are subsequently quantified. Bacteria and metabolites that show statistical differences between control and experimental groups may be involved in treatment efficacy of the disease in question, and accordingly will be targets of interest. These bacteria (potential probiotics) or compounds (potential postbiotics or paraprobiotics), either singly or in a consortium, will then be systematically assessed for their function and mechanism. To achieve this, cutting-edge analyses platforms, such as next generation sequencing (NGS), proteomics, and metabolomics are to be used.

The experimental design of microbiota-based TCM multiomics research is described as follows (Fig. 2): (i) In preparing TCM herbal products such as decoction, strictly follow the instructions for consistency. (ii) Optimally grouping the animals and humans, followed by observing disease progression and development, and finally evaluating the results by statistics. (iii) The composition of gut microbiota and/or their predicted functions of gut microbiome are analyzed by $16 \mathrm{~S}$ rRNA gene sequencing, or shotgun sequencing, respectively, followed by bioinformatics analyses on operational taxonomic units (OTUs), and other functional DNA fragments. (iv) Metabolites will be analyzed by metabolomics combined with other analyses such as metatranscriptomics (for gut microbiota), and epigenetics, transcriptomics/single cell RNA sequencing (scRNA seq), and proteomics (for host). This multiomics approach will work in concert with traditional immunological, physiological, and pathological analyses for holistic results. (v) Associations between and among datasets obtained from control and experimental groups are analyzed to identify parameters with significant differences. Potential enriched or decreased biochemical pathways are established by GO and KEGG pathway analyses. Besides, some databases related to TCM and gut microbiota research including the TCM-Mesh, TCMSP, Traditional Chinese Medicines Integrated Database (TCMID), Compound Reference Database (CRD) and HIT, as well as pathogen-host databases (PHI-base and EHFPI) can be used as references of analytical systems for TCM pharmacology network analysis (Zhang et al., 2020a, b). (v) Isolate selected microbiota bacteria and purify or synthesize novel functional metabolites for further evaluation or validation of their safety and efficacy. (vii) Based on the results obtained, evaluate the possibility of further clinical trials.

Critically, after fermentation of TCM ingredients by microbiota, only important elements involved in the regulation of signaling pathways and diseases amelioration are highlighted. These elements are then selected to develop new treatment strategies. Currently, there are many multiomics-based templates that can be recruited as references for use in TCM and microbiota related studies. For instance, a functional analysis of the gut microbiota ecosystem for better understanding inflammatory bowel disease (IBD) (Lloyd-Price et al., 2019), while the modified ColPortal platform (Esteban-Gil et al., 2019) integrates multiomics studies to understand the relationship between the microbiota and metabolomics in inflammatory bowel disease (IBS) disease model (Liu et al., 2019b). The pipelines described here have the potential to identify novel TCM-based herbal prebiotics, probiotics, and postbiotics for treatment of disease.

\section{PERSPECTIVE}

Recent studies have demonstrated that gut microbiota participate in the metabolism of foods and nutrients and play central roles in the transformation of original TCM herbal components into functional metabolites. Under increasing studies on characterizing the metabolites after administration of various TCM herbs and gut microbiota transformation, together with the help from cutting-edge multiomics research tools, such as the NGS and metabolomics platforms, as well as the advanced bioinformatics analyses, databases, and algorithms, the identification of novel and effective metabolites for safe treatments is possible. Ultimately, a better understanding of the molecular mechanisms TCM function will make great contributions to the effective treatment of the chronic inflammation related diseases.

\section{ACKNOWLEDGEMENTS}

We would like to express our thankfulness for funding provided from CORPD1F0013 and CORPD1J0052 from Chang Gung Memorial Hospital, Microbiota Research Center from Chang Gung University, and the Research Center for Emerging Viral Infections from The Featured Areas Research Center Program within the framework of the Higher Education Sprout Project (MOST109-2634F-182-001, 109-2320-B-030-010, 109-2327-B-182-001).

\section{ABBREVIATIONS}

ADME, absorption/distribution/metabolism/excretion; Ara, arabinose; BAT, brown adipose tissue; BCAAs, branched chain amino acids; CAZymes, carbohydrate active enzymes; CFU, colony-forming units; CKD, chronic kidney disease; CRD, compound Reference Database; C-T-D, component-target-disease; CVD, cardiovascular disease; DAMPS, damage-associated molecular pattern molecules; DSHT, Daesiho-Tang; DST, Du-Shen-Tang; F/B, Firmicutes/Bacteroidetes; Fuc, Fucose; FZHY, Fuzheng Huayu; Gal, galactose; GI, gastrointestinal; Glc, glucose; GO, Gene Ontology; GQD, Gegen Qinlian decoction; GUS, $\beta$-glucosidase; HTS, high-throughput screening; IBD, inflammatory bowel disease; IECs, intestinal epithelial cells; Ile, isoleucine; KEGG, Kyoto Encyclopedia of Genes and Genomes; Leu, leucine; MA, myristoleic acid; Man, mannose; NAFLD, non-alcoholic fatty liver disease; NGS, next generation sequencing; OTUs, operational taxonomic units; PAMPS, pathogenassociated molecular pattern molecules; PD, pharmacodynamics; PK, pharmacokinetics; PS, polysaccharides; PULs, polysaccharide utilization loci; QHD, Qushi Huayu Decoction; Rha, rhamnose; 
SCFAs, short chain fatty acids; scRNA seq, single cell RNA sequencing; Sus, starch utilization system; TCM, Traditional Chinese Medicine; TCMID, Traditional Chinese Medicines Integrated Database; TCMSP, TCM System Pharmacology Database and Analysis Platform; TMA, trimethylamine; TMAO, trimethylamine- $N$-oxide; T-P, target-pathway; UGT, UDPglucuronosyltransferase; Val, valine.

\section{COMPLIANCE WITH ETHICS GUIDELINES}

Tzu-Lung Lin, Chia-Chen Lu, Wei-Fan Lai, Ting-Shu Wu, Jang-Jih Lu, Young-Mao Chen, Chi-Meng Tzeng, Hong-Tao Liu, Hong Wei, and Hsin-Chih Lai declare that they have no conflict of interest.

\section{OPEN ACCESS}

This article is licensed under a Creative Commons Attribution 4.0 International License, which permits use, sharing, adaptation, distribution and reproduction in any medium or format, as long as you give appropriate credit to the original author(s) and the source, provide a link to the Creative Commons licence, and indicate if changes were made. The images or other third party material in this article are included in the article's Creative Commons licence, unless indicated otherwise in a credit line to the material. If material is not included in the article's Creative Commons licence and your intended use is not permitted by statutory regulation or exceeds the permitted use, you will need to obtain permission directly from the copyright holder. To view a copy of this licence, visit http:// creativecommons.org/licenses/by/4.0/.

\section{REFERENCES}

Alexandrov P, Zhai Y, Li W, Lukiw W (2019) Lipopolysaccharidestimulated, NF-kB-, miRNA-146a- and miRNA-155-mediated molecular-genetic communication between the human gastrointestinal tract microbiome and the brain. Folia Neuropathol 57:211-219

Amaretti A, Gozzoli C, Simone M, Raimondi S, Righini L, PerezBrocal V, Garcia-Lopez R, Moya A, Rossi M (2019) Profiling of protein degraders in cultures of human gut microbiota. Front Microbiol 10:2614

Anlu W, Dongcheng C, He Z, Qiuyi L, Yan Z, Yu Q, Hao X, Keji C (2019) Using herbal medicine to target the "microbiota-metabolism-immunity" axis as possible therapy for cardiovascular disease. Pharmacol Res 142:205-222

Bailly C (2019) Irinotecan: 25 years of cancer treatment. Pharmacol Res 148:104398

Baumann U, Bisping B (1995) Proteolysis during tempe fermentation. Food Microbiol 12:39-47

Bayer EA, Lamed R, White BA, Flint HJ (2008) From cellulosomes to cellulosomics. Chem Rec 8:364-377

Bei WJ, Guo J, Wu HY, Cao Y (2012) Lipid-regulating effect of traditional chinese medicine: mechanisms of actions. Evid Based Complement Altern Med 2012:970635

Belcher SM, Cline JM, Conley J, Groeters S, Jefferson WN, Law M, Mackey E, Suen AA, Williams CJ, Dixon D et al (2019) Endocrine disruption and reproductive pathology. Toxicol Pathol 47:10491071

Blachier F, Mariotti F, Huneau JF, Tome D (2007) Effects of amino acid-derived luminal metabolites on the colonic epithelium and physiopathological consequences. Amino Acids 33:547-562

Blaut M, Clavel T (2007) Metabolic diversity of the intestinal microbiota: implications for health and disease. J Nutr 137:751S-755S

Bone E, Tamm A, Hill M (1976) The production of urinary phenols by gut bacteria and their possible role in the causation of large bowel cancer. Am J Clin Nutr 29:1448-1454

Bouhnik Y, Raskine L, Simoneau G, Vicaut E, Neut C, Flourie B, Brouns F, Bornet FR (2004) The capacity of nondigestible carbohydrates to stimulate fecal bifidobacteria in healthy humans: a double-blind, randomized, placebo-controlled, parallel-group, dose-response relation study. Am J Clin Nutr 80:16581664

Boyapati RK, Rossi AG, Satsangi J, Ho GT (2016) Gut mucosal DAMPs in IBD: from mechanisms to therapeutic implications. Mucosal Immunol 9:567-582

Burokas A, Arboleya S, Moloney RD, Peterson VL, Murphy K, Clarke G, Stanton C, Dinan TG, Cryan JF (2017) Targeting the microbiota-gut-brain axis: prebiotics have anxiolytic and antidepressant-like effects and reverse the impact of chronic stress in mice. Biol Psychiatry 82:472-487

Canyelles M, Tondo M, Cedo L, Farras M, Escola-Gil JC, BlancoVaca F (2018) Trimethylamine N-Oxide: a link among diet, gut microbiota, gene regulation of liver and intestine cholesterol homeostasis and HDL function. Int J Mol Sci 19(10):3228

Carqueijeiro I, Langley C, Grzech D, Koudounas K, Papon N, O'Connor SE, Courdavault V (2019) Beyond the semi-synthetic artemisinin: metabolic engineering of plant-derived anti-cancer drugs. Curr Opin Biotechnol 65:17-24

Chang CJ, Lin CS, Lu CC, Martel J, Ko YF, Ojcius DM, Tseng SF, Wu TR, Chen YY, Young JD et al (2015) Ganoderma lucidum reduces obesity in mice by modulating the composition of the gut microbiota. Nat Commun 6:7489

Chang CJ, Lin TL, Tsai YL, Wu TR, Lai WF, Lu CC, Lai HC (2019) Next generation probiotics in disease amelioration. J Food Drug Anal 27:615-622

Chang R (2002) Bioactive polysaccharides from traditional Chinese medicine herbs as anticancer adjuvants. J Altern Complement Med 8:559-565

Chen C, You LJ, Huang Q, Fu X, Zhang B, Liu RH, Li C (2018a) Modulation of gut microbiota by mulberry fruit polysaccharide treatment of obese diabetic $\mathrm{db} / \mathrm{db}$ mice. Food Funct 9:3732-3742

Chen J, Hu Y, Chen L, Liu W, Mu Y, Liu P (2019) The effect and mechanisms of Fuzheng Huayu formula against chronic liver diseases. Biomed Pharmacother 114:108846

Chen L, Krekels EHJ, Verweij PE, Buil JB, Knibbe CAJ, Bruggemann RJM (2020) Pharmacokinetics and pharmacodynamics of posaconazole. Drugs 80:671-695

Chen M, Liao Z, Lu B, Wang M, Lin L, Zhang S, Li Y, Liu D, Liao Q, Xie Z (2018b) Huang-Lian-Jie-Du-Decoction ameliorates hyperglycemia and insulin resistant in association with gut microbiota modulation. Front Microbiol 9:2380 
Chen Q, Xiao S, Li Z, Ai N, Fan X (2015) Chemical and metabolic profiling of Si-Ni decoction analogous formulae by high performance liquid chromatography-mass spectrometry. Sci Rep 5:11638

Chen Y, Yao F, Ming K, Wang D, Hu Y, Liu J (2016) Polysaccharides from traditional chinese medicines: extraction, purification, modification, and biological activity. Molecules 21:1705

Chi X, Wang S, Baloch Z, Zhang H, Li X, Zhang Z, Zhang H, Dong Z, Lu Y, Yu H et al (2019) Research progress on classical traditional Chinese medicine formula Lily Bulb and Rehmannia Decoction in the treatment of depression. Biomed Pharmacother 112:108616

Clemente JC, Ursell LK, Parfrey LW, Knight R (2012) The impact of the gut microbiota on human health: an integrative view. Cell 148:1258-1270

Cockburn DW, Koropatkin NM (2016) Polysaccharide degradation by the intestinal microbiota and its influence on human health and disease. J Mol Biol 428:3230-3252

Conlon MA, Bird AR (2014) The impact of diet and lifestyle on gut microbiota and human health. Nutrients 7:17-44

Creekmore BC, Gray JH, Walton WG, Biernat KA, Little MS, Xu Y, Liu J, Gharaibeh RZ, Redinbo MR (2019) Mouse gut microbiomeencoded beta-glucuronidases identified using metagenome analysis guided by protein structure. Systems 4:e00452-19

Dabek M, McCrae SI, Stevens VJ, Duncan SH, Louis P (2008) Distribution of beta-glucosidase and beta-glucuronidase activity and of beta-glucuronidase gene gus in human colonic bacteria. FEMS Microbiol Ecol 66:487-495

Deng Z, Jing WG, Wang SH, Jiao MJ, Zhang Q, Zhou HY, Zhang J, Liu A (2019) Discussion and research progress in standard decoction of medicinal slices. Zhongguo Zhong Yao Za Zhi 44:242-248

Devillard E, McIntosh FM, Duncan SH, Wallace RJ (2007) Metabolism of linoleic acid by human gut bacteria: different routes for biosynthesis of conjugated linoleic acid. J Bacteriol 189:2566-2570

Devkota S, Wang Y, Musch MW, Leone V, Fehlner-Peach $\mathrm{H}$, Nadimpalli A, Antonopoulos DA, Jabri B, Chang EB (2012) Dietary-fat-induced taurocholic acid promotes pathobiont expansion and colitis in II10-/- mice. Nature 487:104-108

Dey $P$ (2019) Gut microbiota in phytopharmacology: a comprehensive overview of concepts, reciprocal interactions, biotransformations and mode of actions. Pharmacol Res 147:104367

Di S, Wang Y, Han L, Bao Q, Gao Z, Wang Q, Yang Y, Zhao L, Tong $X(2019)$ The intervention effect of traditional Chinese medicine on the intestinal flora and its metabolites in glycolipid metabolic disorders. Evid Based Complement Alternat Med 2019:2958920

Ding Z, Zhong R, Xia T, Yang Y, Xing N, Wang W, Wang Y, Yang B, Sun $X$, Shu $Z$ (2020) Advances in research into the mechanisms of Chinese Materia Medica against acute lung injury. Biomed Pharmacother 122:109706

Esteban-Gil A, Perez-Sanz F, Garcia-Solano J, AlburquerqueGonzalez B, Parreno-Gonzalez MA, Legaz-Garcia MDC, Fernandez-Breis JT, Rodriguez-Braun E, Pimentel P, Tuomisto A et al (2019) ColPortal, an integrative multiomic platform for analysing epigenetic interactions in colorectal cancer. Sci Data 6:255

Feng Q, Gou X-J, Meng S-X, Huang C, Zhang Y-Q, Tang Y-J, Wang W-J, Xu L, Peng J, Hu Y-Y (2013) Qushi Huayu decoction inhibits hepatic lipid accumulation by activating AMP-activated protein kinase in vivo and in vitro. Evid-Based Complement Alterna Med: eCAM 2013:184358

Feng W, Ao H, Peng C, Yan D (2019) Gut microbiota, a new frontier to understand traditional Chinese medicines. Pharmacol Res 142:176-191

Gao K, Yang R, Zhang J, Wang Z, Jia C, Zhang F, Li S, Wang J, Murtaza G, Xie H et al (2018) Effects of Qijian mixture on type 2 diabetes assessed by metabonomics, gut microbiota and network pharmacology. Pharmacol Res 130:93-109

Gao W, Guo SZ, Han LW, Zhang FZ (2016) Analysis of projects funded by NSFC in field of Chinese material medica resource in recent three years. Zhongguo Zhong Yao Za Zhi 41:3696-3701

Gentile CL, Weir TL (2018) The gut microbiota at the intersection of diet and human health. Science 362:776-780

Gerard P (2013) Metabolism of cholesterol and bile acids by the gut microbiota. Pathogens 3:14-24

Ghazalpour A, Cespedes I, Bennett BJ, Allayee H (2016) Expanding role of gut microbiota in lipid metabolism. Curr Opin Lipidol 27:141-147

Han Y, Li C, Huan Y, Sun S, Mu Y, Shen Z (2016) Effects of berberine compatible with stachyose on glucolipid metabolism and gut microbiota in diabetic mice. Chin J Clin Pharmacol 32:1121-1124

Hao YF, Jiang JG (2015) Origin and evolution of China Pharmacopoeia and its implication for traditional medicines. Mini Rev Med Chem 15:595-603

Hu HC, Zheng LT, Yin HY, Tao Y, Luo XQ, Wei KS, Yin LP (2019) A significant association between rhein and diabetic nephropathy in animals: a systematic review and meta-analysis. Front Pharmacol 10:1473

Huang X, Chen W, Yan C, Yang R, Chen Q, Xu H, Huang Y (2019) Gypenosides improve the intestinal microbiota of non-alcoholic fatty liver in mice and alleviate its progression. Biomed Pharmacother 118:109258

Humblot C, Murkovic M, Rigottier-Gois L, Bensaada M, Bouclet A, Andrieux C, Anba J, Rabot S (2007) Beta-glucuronidase in human intestinal microbiota is necessary for the colonic genotoxicity of the food-borne carcinogen 2-amino-3-methylimidazo [4,5-f]quinoline in rats. Carcinogenesis 28:2419-2425

Hussain A, Yadav MK, Bose S, Wang JH, Lim D, Song YK, Ko SG, Kim H (2016) Daesiho-Tang is an effective herbal formulation in attenuation of obesity in mice through alteration of gene expression and modulation of intestinal microbiota. PLoS ONE 11: e0165483

Janeiro MH, Ramirez MJ, Milagro FI, Martinez JA, Solas M (2018) Implication of trimethylamine $\mathrm{N}$-Oxide (TMAO) in disease: potential biomarker or new therapeutic target. Nutrients 10 (10): 1398

Jia W, Xie G, Jia W (2018) Bile acid-microbiota crosstalk in gastrointestinal inflammation and carcinogenesis. Nat Rev Gastroenterol Hepatol 15:111-128

Jiang Y, Liu M, Liu H, Liu S (2020) A critical review: traditional uses, phytochemistry, pharmacology and toxicology of Stephania tetrandra S. Moore (Fen Fang Ji). Phytochem Rev 19:449-489

Jin R, Lin ZJ, Xue CM, Zhang B (2013) An improved associationmining research for exploring Chinese herbal property theory: 
based on data of the Shennong's Classic of Materia Medica. J Integr Med 11:352-365

Kaur H, Bose C, Mande SS (2019) Tryptophan metabolism by gut microbiome and gut-brain-axis: an in silico analysis. Front Neurosci 13:1365

Kikugawa K, Kato T (1988) Formation of a mutagenic diazoquinone by interaction of phenol with nitrite. Food and Chemical Toxicology 26:209-214

Kim DH (2018) Gut microbiota-mediated pharmacokinetics of ginseng saponins. J Ginseng Res 42:255-263

Kim HM, Song Y, Hyun GH, Long NP, Park JH, Hsieh YSY, Kwon SW (2020) Characterization and Antioxidant Activity Determination of Neutral and Acidic Polysaccharides from Panax Ginseng C.A. Meyer. Molecules 25:791

Kim KA, Jung IH, Park SH, Ahn YT, Huh CS, Kim DH (2013) Comparative analysis of the gut microbiota in people with different levels of ginsenoside Rb1 degradation to compound $\mathrm{K}$. PLoS One 8:e62409

Koropatkin NM, Cameron EA, Martens EC (2012) How glycan metabolism shapes the human gut microbiota. Nat Rev Microbiol 10:323-335

Lagier JC, Dubourg G, Million M, Cadoret F, Bilen M, Fenollar F, Levasseur A, Rolain JM, Fournier PE, Raoult D (2018) Culturing the human microbiota and culturomics. Nat Rev Microbiol 16:540-550

Lee JW, Na D, Park JM, Lee J, Choi S, Lee SY (2012) Systems metabolic engineering of microorganisms for natural and nonnatural chemicals. Nat Chem Biol 8:536-546

Leng J, Huang F, Hai Y, Tian H, Liu W, Fang Y, Hu Y, Peng J (2020) Amelioration of non-alcoholic steatohepatitis by Qushi Huayu decoction is associated with inhibition of the intestinal mitogenactivated protein kinase pathway. Phytomedicine 66:153135

Li C, Du X, Liu Y, Liu QQ, Zhi WB, Wang CL, Zhou J, Li Y, Zhang H (2020) A systems pharmacology approach for identifying the multiple mechanisms of action for the Rougui-Fuzi Herb pair in the treatment of cardiocerebral vascular diseases. Evid Based Complement Alternat Med 2020:5196302

Li G, Wang D, Fang S, Xu M (2014) Textual research on Costus root (Aucklandia lappa Decne) in the Sheng nong ben cao jing (Shennong's Classic of Materia Medica). Zhonghua Yi Shi Za Zhi 44:135-137

Li LC, Kan LD (2017) Traditional Chinese medicine for pulmonary fibrosis therapy: Progress and future prospects. J Ethnopharmacol 198:45-63

Li SP, Wu D-T, Lv G-P, Zhao J (2013) Carbohydrates analysis in herbal glycomics. TrAC Trends Anal Chem 52:155-169

Lin CS, Chang CJ, Lu CC, Martel J, Ojcius DM, Ko YF, Young JD, Lai HC (2014) Impact of the gut microbiota, prebiotics, and probiotics on human health and disease. Biomed J 37:259-268

Lin L, Ni B, Lin H, Zhang M, Li X, Yin X, Qu C, Ni J (2015) Traditional usages, botany, phytochemistry, pharmacology and toxicology of Polygonum multiflorum Thunb.: a review. J Ethnopharmacol 159:158-183

Lin R, Liu W, Piao M, Zhu H (2017) A review of the relationship between the gut microbiota and amino acid metabolism. Amino Acids 49:2083-2090
Lin T-L, Shu C-C, Lai W-F, Tzeng C-M, Lai H-C, Lu C-C (2019) Investiture of next generation probiotics on amelioration of diseases-strains do matter. Med Microecol 1-2:100002

Liu C, Li S, Zhang Q, Guo F, Tong M, Martinez M, Wang HH, Zhao Y, Shang D (2019a) Emerging role of chinese herbal medicines in the treatment of pancreatic fibrosis. Am J Chin Med 47:709-726

Liu D, Zhang Y, Liu Y, Hou L, Li S, Tian H, Zhao T (2018a) Berberine modulates gut microbiota and reduces insulin resistance via the TLR4 signaling pathway. Exp Clin Endocrinol Diabetes 126:513520

Liu M, Wang Y, Liu Y, Ruan R (2016) Bioactive peptides derived from traditional Chinese medicine and traditional Chinese food: a review. Food Res Int 89:63-73

Liu S, Si C, Yu Y, Zhao G, Chen L, Zhao Y, Zhang Z, Li H, Chen Y, Min $L$ et al (2019b) Multi-omics analysis of gut microbiota and metabolites in rats with irritable bowel syndrome. Front Cell Infect Microbiol 9:178

Liu Y, Li J, Yu J, Wang Y, Lu J, Shang EX, Zhu Z, Guo J, Duan J (2018b) Disorder of gut amino acids metabolism during CKD progression is related with gut microbiota dysbiosis and metagenome change. J Pharm Biomed Anal 149:425-435

Lloyd-Price J, Arze C, Ananthakrishnan AN, Schirmer M, AvilaPacheco J, Poon TW, Andrews E, Ajami NJ, Bonham KS, Brislawn CJ et al (2019) Multi-omics of the gut microbial ecosystem in inflammatory bowel diseases. Nature 569:655-662

Lu Y-M, Xie J-J, Peng C-G, Wang B-H, Wang K-C, Li L-J (2019) Enhancing clinical efficacy through the gut microbiota: a new field of traditional Chinese medicine. Engineering 5:40-49

Lyu M, Wang YF, Fan GW, Wang XY, Xu SY, Zhu Y (2017) Balancing herbal medicine and functional food for prevention and treatment of cardiometabolic diseases through modulating gut microbiota. Front Microbiol 8:2146

Ma N, Tian Y, Wu Y, Ma X (2017) Contributions of the interaction between dietary protein and gut microbiota to intestinal health. Curr Protein Pept Sci 18:795-808

Madsen L, Myrmel LS, Fjaere E, Liaset B, Kristiansen K (2017) Links between dietary protein sources, the gut microbiota, and obesity. Front Physiol 8:1047

Magee EA, Richardson CJ, Hughes R, Cummings JH (2000) Contribution of dietary protein to sulfide production in the large intestine: an in vitro and a controlled feeding study in humans. Am J Clin Nutr 72:1488-1494

Marques TM, Wall R, O'Sullivan O, Fitzgerald GF, Shanahan F, Quigley EM, Cotter PD, Cryan JF, Dinan TG, Ross RP et al (2015) Dietary trans-10, cis-12-conjugated linoleic acid alters fatty acid metabolism and microbiota composition in mice. $\mathrm{Br} \mathrm{J}$ Nutr 113:728-738

Martel J, Ko YF, Ojcius DM, Lu CC, Chang CJ, Lin CS, Lai HC, Young JD (2017a) Immunomodulatory properties of plants and mushrooms. Trends Pharmacol Sci 38:967-981

Martel J, Ojcius DM, Chang CJ, Lin CS, Lu CC, Ko YF, Tseng SF, Lai HC, Young JD (2017b) Anti-obesogenic and antidiabetic effects of plants and mushrooms. Nat Rev Endocrinol 13:149-160

Martens EC, Lowe EC, Chiang H, Pudlo NA, Wu M, McNulty NP, Abbott DW, Henrissat B, Gilbert HJ, Bolam DN et al (2011) Recognition and degradation of plant cell wall polysaccharides by two human gut symbionts. PLoS Biol 9:e1001221 
Mazzoli R, Pessione E (2016) The neuro-endocrinological role of microbial glutamate and GABA signaling. Front Microbiol 7:1934

Mishima E, Fukuda S, Mukawa C, Yuri A, Kanemitsu Y, Matsumoto Y, Akiyama Y, Fukuda NN, Tsukamoto H, Asaji K et al (2017) Evaluation of the impact of gut microbiota on uremic solute accumulation by a CE-TOFMS-based metabolomics approach. Kidney Int 92:634-645

Neis EP, Dejong CH, Rensen SS (2015) The role of microbial amino acid metabolism in host metabolism. Nutrients 7:2930-2946

Ni M (1995) The yellow emperor's classic of medicine: a new translation of the Neijing Suwen with commentary. Shambhala, Boston and London

Nie C, He T, Zhang W, Zhang G, Ma X (2018) Branched chain amino acids: beyond nutrition metabolism. Int J Mol Sci. 1:1. https://doi. org/10.1136/gutjnl-2019-319114

Nie Q, Chen H, Hu J, Fan S, Nie S (2019) Dietary compounds and traditional Chinese medicine ameliorate type 2 diabetes by modulating gut microbiota. Crit Rev Food Sci Nutr 59:848-863

Peng Y, Li X, Zhang S, Liu Z, Ji J, Wu C, Yang J (2020) Gut microbiota and Chinese medicine syndrome: altered fecal microbiotas in spleen $(\mathrm{Pi})$-deficient patients. J Tradit Chin Med 40:137-143

Portune KJ, Beaumont M, Davila A-M, Tomé D, Blachier F, Sanz Y (2016) Gut microbiota role in dietary protein metabolism and health-related outcomes: the two sides of the coin. Trends Food Sci Technol 57:213-232

Qi LW, Wang CZ, Du GJ, Zhang ZY, Calway T, Yuan CS (2011) Metabolism of ginseng and its interactions with drugs. Curr Drug Metab 12:818-822

Qu W, Liu S, Zhang W, Zhu H, Tao Q, Wang H, Yan H (2019) Impact of traditional Chinese medicine treatment on chronic unpredictable mild stress-induced depression-like behaviors: intestinal microbiota and gut microbiome function. Food Funct 10:58865897

Quan LH, Zhang C, Dong M, Jiang J, Xu H, Yan C, Liu X, Zhou H, Zhang $H$, Chen $L$ et al (2019) Myristoleic acid produced by enterococci reduces obesity through brown adipose tissue activation. Gut. 69(7):1239-1247

Ramesh M, Ahlawat P, Srinivas NR (2010) Irinotecan and its active metabolite, SN-38: review of bioanalytical methods and recent update from clinical pharmacology perspectives. Biomed Chromatogr 24:104-123

Ramirez-Perez O, Cruz-Ramon V, Chinchilla-Lopez P, MendezSanchez N (2017) The role of the gut microbiota in bile acid metabolism. Ann Hepatol 16:s15-s20

Rath S, Heidrich B, Pieper DH, Vital M (2017) Uncovering the trimethylamine-producing bacteria of the human gut microbiota. Microbiome 5:54

Ravcheev DA, Godzik A, Osterman AL, Rodionov DA (2013) Polysaccharides utilization in human gut bacterium Bacteroides thetaiotaomicron: comparative genomics reconstruction of metabolic and regulatory networks. BMC Genomics 14:873

Ru J, Li P, Wang J, Zhou W, Li B, Huang C, Li P, Guo Z, Tao W, Yang $Y$ et al (2014) TCMSP: a database of systems pharmacology for drug discovery from herbal medicines. J Cheminform 6:13

Santa HSD, Romão PRT, Sovrani V, Oliveira FR, Peres A, Monteiro MC (2014) Dietary polysaccharidesand Immune Modulation. In:
Ramawat KG, Mérillon J-M (eds) Polysaccharides: bioactivity and biotechnology. Springer International Publishing, Cham, pp 1-24

Schachter J, Martel J, Lin CS, Chang CJ, Wu TR, Lu CC, Ko YF, Lai HC, Ojcius DM, Young JD (2018) Effects of obesity on depression: a role for inflammation and the gut microbiota. Brain Behav Immun 69:1-8

Schoeler M, Caesar R (2019) Dietary lipids, gut microbiota and lipid metabolism. Rev Endocr Metab Disord 20:461-472

Schwiertz A, Taras D, Schafer K, Beijer S, Bos NA, Donus C, Hardt PD (2010) Microbiota and SCFA in lean and overweight healthy subjects. Obesity (Silver Spring) 18:190-195

Sharon G, Garg N, Debelius J, Knight R, Dorrestein PC, Mazmanian SK (2014) Specialized metabolites from the microbiome in health and disease. Cell Metab 20:719-730

Simpson HL, Campbell BJ (2015) Review article: dietary fibremicrobiota interactions. Aliment Pharmacol Ther 42:158-179

Sun SS, Wang K, Ma K, Bao L, Liu HW (2019) An insoluble polysaccharide from the sclerotium of Poria cocos improves hyperglycemia, hyperlipidemia and hepatic steatosis in ob/ob mice via modulation of gut microbiota. Chin J Nat Med 17:3-14

Sun $Y$ (2011) Structure and biological activities of the polysaccharides from the leaves, roots and fruits of Panax ginseng C.A. Meyer: an overview. Carbohydrate Polymers - CARBOHYD POLYM 85:490-499

Tang D, Kang R, Coyne CB, Zeh HJ, Lotze MT (2012) PAMPs and DAMPs: signal 0 s that spur autophagy and immunity. Immunol Rev 249:158-175

Teijaro CN, Adhikari A, Shen B (2019) Challenges and opportunities for natural product discovery, production, and engineering in native producers versus heterologous hosts. J Ind Microbiol Biotechnol 46:433-444

Thursby E, Juge N (2017) Introduction to the human gut microbiota. Biochem J 474:1823-1836

Tong X, Xu J, Lian F, Yu X, Zhao Y, Xu L, Zhang M, Zhao X, Shen J, Wu $S$ et al (2018) Structural alteration of gut microbiota during the amelioration of human type 2 diabetes with hyperlipidemia by metformin and a traditional Chinese herbal formula: a multicenter, randomized, Open label clinical trial. mBio. 9(3):e02392-17

Tremaroli V, Backhed F (2012) Functional interactions between the gut microbiota and host metabolism. Nature 489:242-249

Tsai YL, Lin TL, Chang CJ, Wu TR, Lai WF, Lu CC, Lai HC (2019) Probiotics, prebiotics and amelioration of diseases. J Biomed Sci 26:3

Tzianabos AO (2000) Polysaccharide immunomodulators as therapeutic agents: structural aspects and biologic function. Clin Microbiol Rev 13:523-533

van Duynhoven J, Vaughan EE, Jacobs DM, Kemperman RA, van Velzen EJ, Gross G, Roger LC, Possemiers S, Smilde AK, Dore J et al (2011) Metabolic fate of polyphenols in the human superorganism. Proc Natl Acad Sci USA 108(Suppl 1):4531-4538

Velenosi TJ, Hennop A, Feere DA, Tieu A, Kucey AS, Kyriacou P, McCuaig LE, Nevison SE, Kerr MA, Urquhart BL (2016) Untargeted plasma and tissue metabolomics in rats with chronic kidney disease given AST-120. Sci Rep 6:22526

Wahlstrom A, Sayin SI, Marschall HU, Backhed F (2016) Intestinal crosstalk between bile acids and microbiota and its impact on host metabolism. Cell Metab 24:41-50 
Wan JY, Wang CZ, Zhang QH, Liu Z, Musch MW, Bissonnette M, Chang EB, Li P, Qi LW, Yuan CS (2017) Significant difference in active metabolite levels of ginseng in humans consuming Asian or Western diet: the link with enteric microbiota. Biomed Chromatogr. https://doi.org/10.1002/bmc.3851

Wang B, Yao M, Lv L, Ling Z, Li L (2017a) The human microbiota in health and disease. Engineering 3:71-82

Wang C, Yin Y, Cao X, Li X (2016) Effects of Maydis stigma polysaccharide on the intestinal microflora in type-2 diabetes. Pharm Biol 54:3086-3092

Wang JH, Bose S, Lim SK, Ansari A, Chin YW, Choi HS, Kim H (2017b) Houttuynia cordata facilitates metformin on ameliorating insulin resistance associated with gut microbiota alteration in OLETF rats. Genes (Basel) 8:239

Wang JH, Bose S, Shin NR, Chin YW, Choi YH, Kim H (2018a) Pharmaceutical impact of houttuynia cordata and metformin combination on high-fat-diet-induced metabolic disorders: link to intestinal microbiota and metabolic endotoxemia. Front Endocrinol (Lausanne) 9:620

Wang L, Liu QM, Sung BH, An DS, Lee HG, Kim SG, Kim SC, Lee $\mathrm{ST}$, Im WT (2011) Bioconversion of ginsenosides $\mathrm{Rb}(1), \mathrm{Rb}(2)$, $\mathrm{Rc}$ and $\mathrm{Rd}$ by novel beta-glucosidase hydrolyzing outer 3-O glycoside from Sphingomonas sp. 2F2: cloning, expression, and enzyme characterization. J Biotechnol 156:125-133

Wang Q, Kuang H, Su Y, Sun Y, Feng J, Guo R, Chan K (2013) Naturally derived anti-inflammatory compounds from Chinese medicinal plants. J Ethnopharmacol 146:9-39

Wang R, Zang P, Chen J, Wu F, Zheng Z, Ma J, Yang C, Du H (2018b) Gut microbiota play an essential role in the antidiabetic effects of rhein. Evid Based Complement Alternat Med 2018:6093282

Wei X, Tao J, Xiao S, Jiang S, Shang E, Zhu Z, Qian D, Duan J (2018) Xiexin Tang improves the symptom of type 2 diabetic rats by modulation of the gut microbiota. Sci Rep 8:3685

Wu TR, Lin CS, Chang CJ, Lin TL, Martel J, Ko YF, Ojcius DM, Lu CC, Young JD, Lai HC (2019) Gut commensal Parabacteroides goldsteinii plays a predominant role in the anti-obesity effects of polysaccharides isolated from Hirsutella sinensis. Gut 68:248262

Wu XM, Tan RX (2019) Interaction between gut microbiota and ethnomedicine constituents. Nat Prod Rep 36:788-809

Xu J, Lian F, Zhao L, Zhao Y, Chen X, Zhang X, Guo Y, Zhang C, Zhou $Q$, Xue $Z$ et al (2015) Structural modulation of gut microbiota during alleviation of type 2 diabetes with a Chinese herbal formula. ISME J 9:552-562

Xu JD, Mao Q, Shen H, Zhu LY, Li SL, Yan R (2013) Ultra-high performance liquid chromatography coupled with photo-diode array and quadrupole/time-of-flight mass spectrometry based chemical profiling approach to evaluate the influence of preparation methods on the holistic quality of Qiong-Yu-Gao, a traditional complex herbal medicine. J Chromatogr A 1304:154168

Xu M, Yue RS, Yang MY, Yang X, Wu TC, Li JN (2018) Effects of Banxia Xiexin Decoction on intestinal flora and inflammatory factors of diabetic gastroparesis rats. Chinese Traditional and Herbal Drugs 49:3056-3061
Yan Q (2018) Neuroimmune imbalances and Yin-Yang dynamics in stress, anxiety, and depression. Methods Mol Biol 1781:77-85

Yan Y, Chai CZ, Wang DW, Yue XY, Zhu DN, Yu BY (2013) HPLCDAD-Q-TOF-MS/MS analysis and HPLC quantitation of chemical constituents in traditional Chinese medicinal formula Ge-Gen Decoction. J Pharm Biomed Anal 80:192-202

Yang M, Lao L (2019) Emerging applications of metabolomics in traditional Chinese medicine treating hypertension: biomarkers. Pathways and More. Front Pharmacol 10:158

Yang M, Sun J, Lu Z, Chen G, Guan S, Liu X, Jiang B, Ye M, Guo DA (2009) Phytochemical analysis of traditional Chinese medicine using liquid chromatography coupled with mass spectrometry. J Chromatogr A 1216:2045-2062

Yang YS, Zhang T, Yu SC, Ding Y, Zhang LY, Qiu C, Jin D (2011) Transformation of geniposide into genipin by immobilized betaglucosidase in a two-phase aqueous-organic system. Molecules 16:4295-4304

Ying M, Zheng B, Yu Q, Hou K, Wang H, Zhao M, Chen Y, Xie J, Nie S, Xie M (2020) Ganoderma atrum polysaccharide ameliorates intestinal mucosal dysfunction associated with autophagy in immunosuppressed mice. Food Chem Toxicol 138:111244

Yokoi T, Narita M, Nagai E, Hagiwara H, Aburada M, Kamataki T (1995) Inhibition of UDP-glucuronosyltransferase by aglycons of natural glucuronides in kampo medicines using $\mathrm{SN}-38$ as a substrate. Jpn J Cancer Res 86:985-989

Yu J, Guo J, Tao W, Liu P, Shang E, Zhu Z, Fan X, Shen J, Hua Y, Zhu KY et al (2018a) Gancao-Gansui combination impacts gut microbiota diversity and related metabolic functions. J Ethnopharmacol 214:71-82

Yu Y, Li Z, Guo R, Qian J, Zhang H, Zhang J, Zhao X, Wang S, Wang $Y(2019 a)$ Ononin, sec-O-beta-d-glucosylhamaudol and astragaloside I: antiviral lead compounds identified via high throughput screening and biological validation from traditional Chinese medicine Zhongjing formulary. Pharmacol Res 145:104248

Yu Y, Raka F, Adeli K (2019b) The role of the gut microbiota in lipid and lipoprotein metabolism. J Clin Med 8(12):2227

Yu Y, Shen M, Song Q, Xie J (2018b) Biological activities and pharmaceutical applications of polysaccharide from natural resources: a review. Carbohydr Polym 183:91-101

Yuan Y, Zheng J, Wang M, Li Y, Ruan J, Zhang H (2016) Metabolic activation of rhein: insights into the potential toxicity induced by rhein-containing herbs. J Agric Food Chem 64:5742-5750

Yue SJ, Wang WX, Yu JG, Chen YY, Shi XQ, Yan D, Zhou GS, Zhang L, Wang CY, Duan JA et al (2019) Gut microbiota modulation with traditional Chinese medicine: a system biologydriven approach. Pharmacol Res 148:104453

Zhang B, Yue R, Chen Y, Yang M, Huang X, Shui J, Peng Y, Chin J (2019a) Gut microbiota, a potential new target for Chinese herbal medicines in treating diabetes mellitus. Evid Based Complement Alternat Med 2019:2634898

Zhang D, Chia C, Jiao X, Jin W, Kasagi S, Wu R, Konkel JE, Nakatsukasa H, Zanvit P, Goldberg N et al (2017) D-mannose induces regulatory $T$ cells and suppresses immunopathology. Nat Med 23:1036-1045

Zhang D, Yang L, Yang LX, Wang MY, Tu YY (2007) Determination of artemisinin, arteannuin $B$ and artemisinic acid in Herba 
Artemisiae Annuae by HPLC-UV-ELSD. Yao Xue Xue Bao 42:978-981

Zhang D, Zhang Y, Gao Y, Chai X, Pi R, Chan G, Hu Y (2020a) Translating traditional herbal formulas into modern drugs: a network-based analysis of Xiaoyao decoction. Chin Med 15:25

Zhang ND, Han T, Huang BK, Rahman K, Jiang YP, Xu HT, Qin LP, Xin HL, Zhang QY, Li YM (2016) Traditional Chinese medicine formulas for the treatment of osteoporosis: Implication for antiosteoporotic drug discovery. J Ethnopharmacol 189:61-80

Zhang R, Gao X, Bai H, Ning K (2020b) Traditional Chinese medicine and gut microbiome: their respective and concert effects on healthcare. Front Pharmacol 1:1. https://doi.org/10. 3389/fphar.2020.00538

Zhang T, Yang Y, Liang Y, Jiao X, Zhao C (2018) Beneficial effect of intestinal fermentation of natural polysaccharides. Nutrients 10 (8):55

Zhang WJ, Wang S, Kang CZ, Lv CG, Zhou L, Huang LQ, Guo LP (2020c) Pharmacodynamic material basis of traditional Chinese medicine based on biomacromolecules: a review. Plant Methods 16:26

Zhang YL, Cai LT, Qi JY, Lin YZ, Dai YC, Jiao N, Chen YL, Zheng L, Wang BB, Zhu LX et al (2019b) Gut microbiota contributes to the distinction between two traditional Chinese medicine syndromes of ulcerative colitis. World J Gastroenterol 25:3242-3255

Zhao CQ, Zhou Y, Ping J, Xu LM (2014) Traditional Chinese medicine for treatment of liver diseases: progress, challenges and opportunities. J Integr Med 12:401-408

Zhou SS, Xu J, Zhu H, Wu J, Xu JD, Yan R, Li XY, Liu HH, Duan SM, Wang $Z$ et al (2016) Gut microbiota-involved mechanisms in enhancing systemic exposure of ginsenosides by coexisting polysaccharides in ginseng decoction. Sci Rep 6:22474

Zhu KX, Nie SP, Tan LH, Li C, Gong DM, Xie MY (2016) A polysaccharide from ganoderma atrum improves liver function in type 2 diabetic rats via antioxidant action and short-chain fatty acids excretion. J Agric Food Chem 64:1938-1944

Zmora N, Suez J, Elinav E (2019) You are what you eat: diet, health and the gut microbiota. Nat Rev Gastroenterol Hepatol 16:35-56 